\title{
Food Messages Adolescents See Daily on Social Media: A Diary Study
}

\section{Abstract}

Billions are spent yearly on food marketing that influences unhealthy eating preferences and habits, and contributes to the development of obesogenic environments worldwide. Recent social media advancements have provided food marketers with platforms to readily reach out to many especially younger consumers. Adolescents seem vulnerable to food marketing as they have increased impulsive decision-making and decreased inhibitory-control as compared to adults. Yet, they are becoming independent from their parents and relying on unmonitored (social) media. Despite the presumed influence of social media marketing, little is known about the type of marketing strategies used on social media to influence adolescent eating.

Aim: The aim of this study is to explore the food messages adolescents (12-18 years old) encounter when using social media.

Methods: A diary study was carried out with 12-18 year-old Belgian adolescents $(\mathrm{N}=20)$ who took screenshots of food images they encountered on their social media during a one-week period. Participants also completed surveys assessing social media use, image source, marketing strategy, etc. Five-hundred food images were gathered and preliminary analyzed for their content. A coding book, developed based on a literature review and a pilot, will guide two authors in coding the images. The codes will be checked for agreement and discrepancies will be resolved among the research team. Finally, the codes will be analyzed to determine food marketing strategies employed and eating social norms portrayed.

Results: The most commonly reported social media were Instagram, Snapchat, Twitter, and Facebook. Preliminary findings showed that adolescents often encounter branded food images through peers, influencers and food brands, majority of which are paid or word-of-mouth food marketing. The images were mostly promoting non-core foods that are energy-dense and poor in nutrients, especially branded soft drinks and fried foods. An ongoing qualitative analysis of the gathered images aims to conclude with detailed frequencies and relationships between the different study measures assessed. These findings will be elaborated on during the presentation.

Discussion: This research provides an in-depth understanding of the social media messages adolescents encounter daily and sheds light on food norms typically communicated on social media by marketers, peers, and influencers. Such findings identify prominent social media food messages that must be tested for their persuasive nature, providing insights for future research that aims to assesses the effects of social media food marketing on adolescent eating. Finally, we call for policies that address current social media marketing strategies targeting adolescents.

\section{Conflict of Interest}

There is no conflict of interest. 